\title{
Effects of TiLOOP Bra Mesh on Radiotherapy Dose Distribution
}

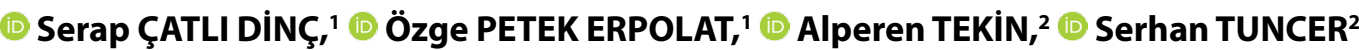 \\ 'Department of Radiation Oncology, Gazi University School of Medicine, Ankara-Turkey \\ ${ }^{2}$ Department of Plastic Surgery, Gazi University School of Medicine, Ankara-Turkey
}

\begin{abstract}
OBJECTIVE
The TiLOOP Bra mesh is a breast-implant-surrounding material that supports the pectoralis muscle and keeps the implant stable in the subpectoral area during breast reconstruction surgery. This study aimed to investigate the dosimetric effect of TiLOOP Bra mesh on dose distribution of radiotherapy in patients requiring postoperative treatment.
\end{abstract}

\section{METHODS}

The metal oxide semiconductor field effect transistor (MOSFET) and nanoDot optically stimulated luminescence dosimeter (OSLD) were used for dose measurements at different depths in solid phantoms. The measurements were performed above and below the mesh, and at $1 \mathrm{~cm}$ deep to the mesh for $6 \mathrm{MV}$ photon energy. The relative dose differences were obtained by measuring doses using the dosimeters and comparing the results with calculated values in Eclipse TPS (Treatment Planning System). The relative dose differences between the cases where the mesh had been present and where the mesh had been removed were evaluated.

\section{RESULTS}

The results were found less than $1 \%$. The findings showed that the TiLOOP Bra mesh used in breast surgery did not affect the dose calculations for radiotherapy. In addition, there were no metallic artifacts on computed tomography image.

\section{CONCLUSION}

Therefore, the quality of the computed tomography image was not affected by the TiLOOP Bra mesh, and it was not necessary to correct the artifact and change the HU (Hounsfield Unit) value in TPS.

Keywords: Dosimetry; MOSFET; OSLD; radiotherapy dose distribution; TiLOOP Bra mesh.

Copyright $\odot$ 2019, Turkish Society for Radiation Oncology

\section{Introduction}

Today, breast reconstruction is an integral part of breast cancer treatment; and implant-based reconstruction is the most commonly preferred technique worldwide. Different alloplastic materials are used in implant-based reconstruction to support pectoralis major muscle and provide an additional layer over the implant. The TiLOOP Bra mesh is one of the materials that have been used for these purposes in breast reconstruction surgery.[1] The titanized polypropylene mesh is an implant-surrounding material that supports the pectoralis muscle and keeps the implant stable in the subpectoral area. High atomic number and density of implants lead to major problems in providing an accurate dose distribution in radiotherapy (RT) and 
contouring tumors and organs caused by the artifact (AAPM-85 report).[2] When a high-density metallic implant is placed in a medium, it can cause artifacts on computed tomography (CT) scans. These artifacts result in uncertainty while calculating dose in TPS. Eventually, this uncertainty may result in significant underdosing to the target volume or overdosing to normal tissue.[3] Therefore, it is recommended that treatment fields should not include prostheses; however, this is not always possible, and the accuracy of the TPS used should be well known in the presence of metallic inhomogeneities.[3,4] Many studies in the literature have studied titanium implants.[3-9] However, studies with the TiLOOP Bra meshes for patients with breast cancer are limited in the literature. Camacho et al evaluated the dosimetric effect of the presence of a TiLOOP Bra mesh on breast RT and radiographic imaging. [10] Cho et al investigated the dosimetric effect of Ti-mesh on the proton beam by measuring the lateral dose profile of the proton beam using EBT3 film and compared the result with the calculated value in TPS.[11] Additionally, Patone et al studied the effect of Ti neurosurgical mesh in a patient treated with 6 and $18 \mathrm{MV}$ photon beams.[12] Rakowski et al reported that due to the existence of a Ti neurosurgical mesh, dose perturbations were found after using MV photon beams.[13]

This study aimed to investigate the dosimetric effect of TiLOOP Bra mesh on RT dose distribution in patients requiring postoperative treatment. The EBT3 radiochromic film was frequently used in previous studies. However, new dosimetric systems such as MOSFET and nanoDot OSLD, which are more sensitive in dose measurements, have not been well studied. The results of dosimetric measurements with MOSFET and nanoDot OSLD and Eclipse TPS values were compared to each other and to those that was previously reported.

\section{Materials and Methods}

TiLOOP Bra is a polypropylene mesh with a submillimeter thickness, totally coated with a very thin (30$50 \mathrm{~nm}$ ) layer of titanium [10] (Fig. 1). The diameter of each pore is $1.0 \mathrm{~mm}$. The TiLOOP Bra mesh has three different sizes: small, medium, and large.

A $5 \times 5 \mathrm{~cm}^{2}$ sample was obtained from medium-size TiLOOP Bra mesh. It had a total thickness of $0.20 \mathrm{~mm}$, and the titanium-coated layer was $0.015 \%-0.025 \%$ of the total mesh thickness. The geometry of solid water phantom was a $30 \times 30 \times 30 \mathrm{~cm}^{3}$, and the TiLOOP Bra mesh was placed at a depth of $5 \mathrm{~cm}$ (Fig. 2).

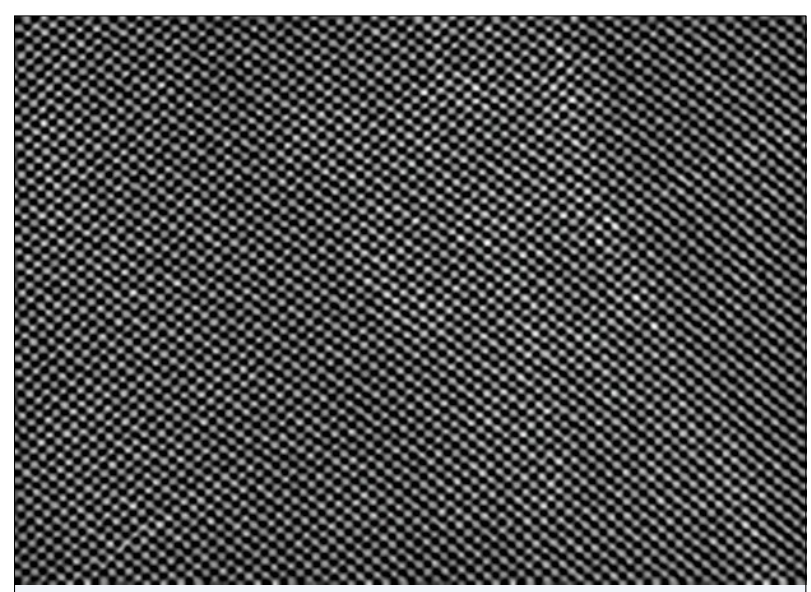

Fig. 1. TiLOOP Bra mesh.

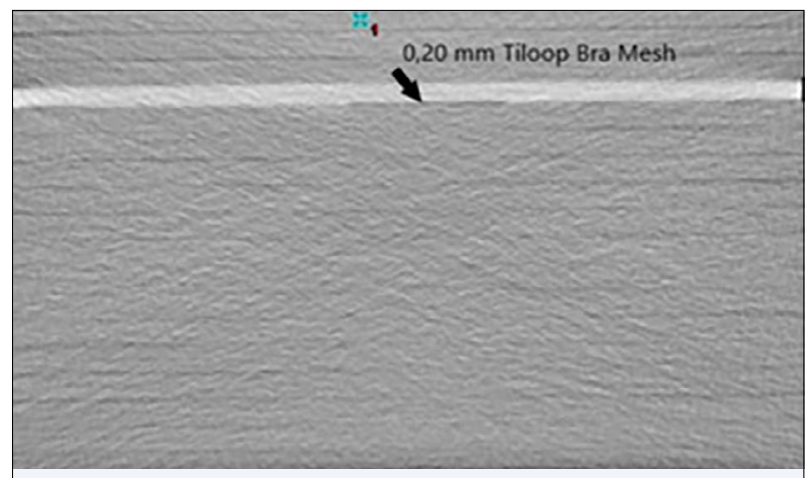

Fig. 2. TiLOOP Bra mesh was placed at a depth of $5 \mathrm{~cm}$ of solid water phantom.

This depth was chosen to avoid the build-up region $(1.5 \mathrm{~cm})$. In clinical practice, the mesh is located within the first centimeters deep to the skin. The phantom was scanned using CT to create a crosssectional image. The CT images of phantoms were obtained for a thickness of $1.5 \mathrm{~mm}$. The images were electronically loaded to TPS. The Eclipse TPS v.8.6.15 (Varian Medical Systems, Palo Alto, CA) was used for dose calculations. This system consists of integrated imaging and 3D dose calculation systems, and can be used for 3D conformal RT. The TPS utilizes a single pencil beam model in conjunction with one of three inhomogeneity correction methods: the Batho power law, the modified Batho (MB), and equivalent tissue air ratio. The dose value calculated in a water-equivalent material is multiplied by inhomogeneity correction factors calculated by the above methods. In this study, because the MB inhomogeneity correction method implemented on the TPS is used in our clinic routine, the pencil beam model was used for dose calculations. The Hounsfield Unit (HU) and electron 
density value of mesh were not changed for calculations. The dose calculation grid size was $0.125 \mathrm{~cm}$, which was the smallest available in TPS.

Since $6 \mathrm{MV}$ energy is mostly preferred in breast cancer RT, the dose plans were obtained by using $6 \mathrm{MV}$ $\mathrm{X}$-ray beam. Depth doses were calculated on the phantom for a source-skin distance (SSD) of $100 \mathrm{~cm}$ and a $10 \times 10 \mathrm{~cm}^{2}$ field (Fig. 3). All doses were normalized by the dose at $Z=1.5 \mathrm{~cm}$.

A dose of $200 \mathrm{cGy}$ was prescribed at $5 \mathrm{~cm}$ depth at where the dosimeters were placed. MOSFET and nanoDot OSLD were used for dose measurements at different depths in solid phantoms.

Initially, the MOSFET calibration was performed. Pre-calculated time (or MU) for $100 \mathrm{cGy}$ for $6 \mathrm{MV}$ photon energy at $5 \mathrm{~cm}$ depth was measured by using ion chamber in solid phantom (i.e., change in the threshold voltage VTH were recorded at the same depth). With these measurements, the calibration factor $(\mathrm{cGy} / \mathrm{mV})$ was obtained. Also, nanoDot OSLD dosimeters were calibrated at $5 \mathrm{~cm}$ depth for $6 \mathrm{MV}$ photon energy.

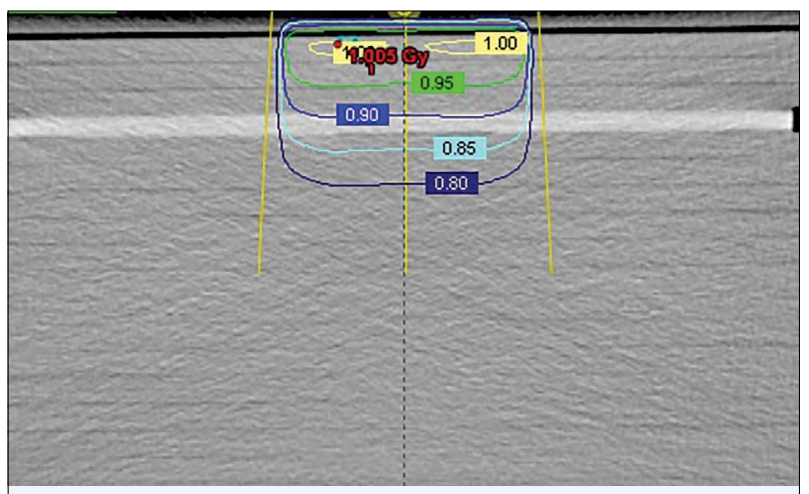

Fig. 3. The depth doses for a source-skin distance (SSD) of $100 \mathrm{~cm}$ and a $10 \times 10 \mathrm{~cm}^{2}$ field.

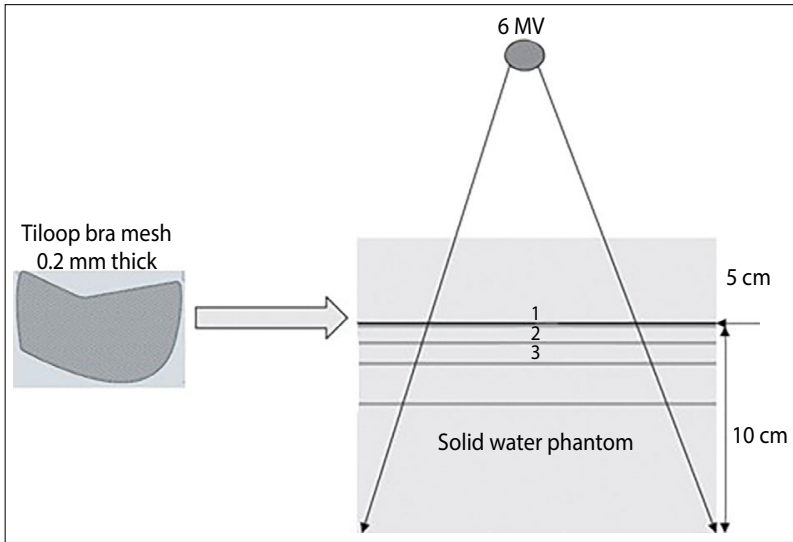

Fig. 4. The schematic of setup for measurements.
After that, three measurements were performed above (1), below (2), and at $1 \mathrm{~cm}$ depth (3) under the mesh, using these calibration methods (Fig. 4). The mean values of the absorbed dose of three measurements were calculated.

Finally, relative dose difference between the absorbed doses with and without mesh and relative difference between the TPS dose values and dosimeter measurements were determined by these formulas:

$$
\begin{aligned}
& \text { Relative difference }=\left[\left(\text { Dose }_{\text {with mesh }}-\text { Dose }_{\text {without mesh }}\right) /\right. \\
& \left.\mathrm{D}_{\text {without mesh }}\right] \times 100 \text { (1) } \\
& \text { Relative difference }=\left[\left(\text { Dose }_{\text {measurements }}-\mathrm{D}_{\mathrm{TPS}}\right) /\right. \\
& \mathrm{D}_{\mathrm{TPS}} \mathrm{C} \times 100 \quad(2) \quad
\end{aligned}
$$

\section{Results}

This study investigated the dosimetric effect of TiLOOP Bra mesh on RT dose. The mean values of the absorbed dose of three measurements (above, below, and at $1 \mathrm{~cm}$ depth under the mesh) were obtained for MOSFET, nanoDot OSLD, and TPS. Using these mean values, the dose differences between the measurements with and without the presence of mesh at three different depths were found less than $1 \%$. However, at $1 \mathrm{~cm}$ depth under the mesh, the dose difference was $2.4 \%$ for nanoDot OSLD technique. The results are presented in Table 1. Camacho et al evaluated the impact of TiLOOP Bra mesh in dose delivery of breast RT. The dosimetric effects had been measured for three photon beam energies (1.25 MeV, $6 \mathrm{MV}$, and $18 \mathrm{MV}$ ), using radiochromic film placed at three different depths. They found that the relative absorbed dose differences with and without the mesh were less than $1 \%$, which was similar to our results.

Table 1 The results of measurements and TPS calculations

$6 \mathrm{MV} \quad \begin{gathered}\text { Dose (cGy), } \\ \text { with mesh }\end{gathered} \quad \begin{gathered}\text { Dose (cGy), } \\ \text { without mesh }\end{gathered} \quad \begin{gathered}\text { Difference } \\ (\%)\end{gathered}$

\begin{tabular}{llll}
\hline MOSFET & & & \\
Above & 183 & 182 & 0.5 \\
Below & 180 & 180 & 0.0 \\
$1 \mathrm{~cm}$ depth & 162 & 162 & 0.0 \\
OSLD & & & \\
Above & 174 & 175 & 0.6 \\
Below & 173 & 174 & 0.5 \\
$1 \mathrm{~cm}$ depth & 163 & 167 & 2.4 \\
TPS & & & \\
Above & 176 & 175 & 0.6 \\
Below & 175 & 174 & 0.6 \\
$1 \mathrm{~cm}$ depth & 169 & 168 & 0.6
\end{tabular}


Table 2 The dose differences between TPS results with measurements

\begin{tabular}{lcccc}
$\mathbf{6}$ MV & $\begin{array}{c}\text { MOSFET-TPS } \\
\text { with mesh }\end{array}$ & $\begin{array}{c}\text { Difference \% } \\
\text { without mesh }\end{array}$ & $\begin{array}{c}\text { OSLD-TPS } \\
\text { with mesh }\end{array}$ & $\begin{array}{c}\text { Difference \% } \\
\text { without mesh }\end{array}$ \\
\hline Above & 3.9 & 4.0 & 1.1 & 0.0 \\
Below & 2.9 & 3.4 & 0.0 & 0.0 \\
1 cm depth & 3.6 & 3.6 & 0.6 & 0.5
\end{tabular}

Additionally, the dose differences between those of two dosimetric techniques were compared to the values that were obtained from TPS (Table 2). The absorbed dose differences between MOSFET and TPS on the presence of mesh were $3.9 \%, 2.9 \%$, and $3.6 \%$ above, below, and at $1 \mathrm{~cm}$ depth under the mesh, respectively. As a result of comparing the dose with MOSFET measurements and TPS calculations without presence of mesh, the absorbed dose differences were $4.0 \%, 3.4 \%$, and $3.6 \%$ above, below, and at $1 \mathrm{~cm}$ depth, respectively. The comparison of the absorbed doses using nanoDot OSLD measurement to TPS calculations on the presence of mesh were $1.1 \%, 0.0 \%$, and $0.6 \%$ above, below, and at $1 \mathrm{~cm}$ depth under the mesh, respectively. The absorbed dose difference without mesh using OSLD, the dose differences has resulted to be less than $0.5 \%$.

Cho et al investigated the dosimetric effect of TiLOOP mesh on the proton beam.[11] They measured the lateral dose profile of the proton using EBT3 film and compared the results with the calculated values in TPS. The results showed no dose reduction in the TiLOOP mesh region and showed a dose profile very similar to that of TPS. The difference between that experimental study and our results can be due to the variability of either different kind of beam or sensitivity, experimental conditions, and setup errors among the dosimetric techniques. The dose differences in our study were less than $5 \%$. According to dosimetric protocols, which recommend that the differences in the dose should be bellow of 5\% [14], the difference found in the study could be considered negligible in clinical practice.

\section{Discussion}

Our findings showed that the presence of the mesh does not appreciably perturb the dose distribution. The implants can cause significant attenuation in the absorbed dose at points beyond the implants. Catli et al found that the decrease in dose $19.9 \%$ at a distance of $0.5 \mathrm{~cm}$ behind the $2 \mathrm{~cm}$ thick titanium plate and the decrease in dose $14.8 \%$ at a distance of $0.5 \mathrm{~cm}$ behind the $1 \mathrm{~cm}$ thick titanium plate.[3-4] Shimozato et al also showed the decrease in dose behind a thin titanium plate (thickness, $0.05 \mathrm{~cm}$ ). [9] The decrease in dose may vary due to thickness of plate, and the dose behind the implant decreases with increasing thickness. This study's findings showed that there was no significant decrease in dose behind the TiLOOP Bra mesh for 6 MV. The titanium-coated mesh is a very thin material compared to titanium plates. In addition, the number and the size of the pores on the mesh were probably the reasons for minimal attenuation behind the implant.

Furthermore, no increase in dose due to the backscatter of electrons was seen within $5 \mathrm{~cm}$ of the phantom from the surface. Catli et al found $8.4 \%$ of dose increase in tissue at a distance of $5 \mathrm{~mm}$ in front of the titanium ( $2 \mathrm{~cm}$ thickness) due to the backscatter of electrons.[3] Shimozato et al also showed dose increases in front of the thin titanium plate.[9] In contrast, Camacho et al showed that there was no increase in dose due to electron backscatter from the TiLOOP Bra mesh.[10] They found that the mesh does not disturb the dosimetry of a typi $\neg$ cal RT treatment. Additionally, Patone et al studied the effect of TiLOOP Bra mesh in a patient treated with 6 and $18 \mathrm{MV}$ photon beams. They concluded that the dosimetric impact of a $0.4 \mathrm{~mm}$ mesh is negligible and does not require modification in treatment parameters.[12] The materials with high atomic number like titanium plate might cause more scattering, as reported by Das and Khan.[15] However, in this study, the titani-

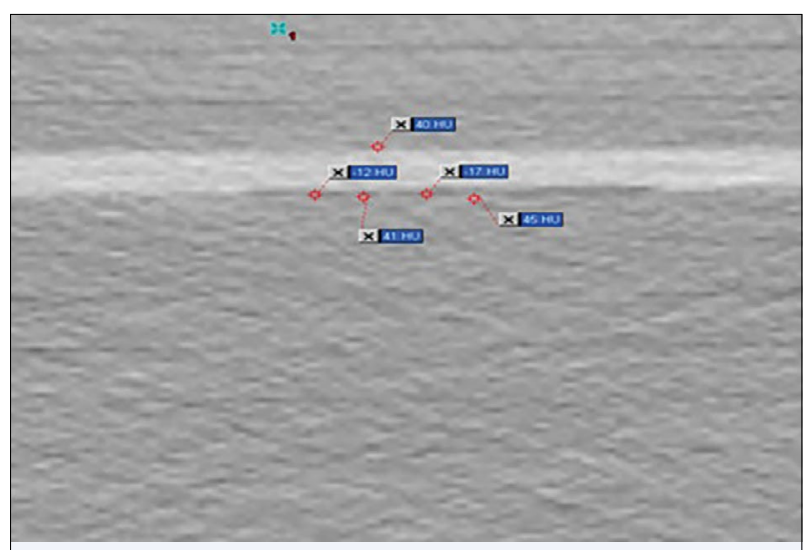

Fig. 5. The HU values of TiLOOP Bra mesh. No metallic artifacts are observed. 
um-coated layer was $0.015 \%-0.025 \%$ of the total mesh thickness. Therefore, the titanium layer was very thin, the attenuation effect was expected to be minimal.

The metal implants should be contoured on CT images; actual electron density and $\mathrm{HU}$ values should be identified on the TPS. However, in this study, the TiLOOP Bra mesh did not cause streak artifacts on CT images. There were no high $\mathrm{HU}$ values, as expected for high-density materials like titanium. The TiLOOP Bra mesh behaved like air on CT images as is shown in Figure 5. While the $\mathrm{HU}$ value of solid water phantom were around 30, the average HU value of mesh was found as -1 . Due to its minimal thickness, the mesh placed between the solid water phantoms could not be appreciated on the image. Therefore, a relative electron density and HU value was not manually assigned to the appropriately contoured volume of the mesh and, the HU value of mesh was not changed in TPS calculations. Its influence on the quality of the CT scan required for planning was negligible, which was also recommended by Camacho et al.[10]

\section{Conclusion}

This study investigated the influence of the titanized mesh TiLOOP Bra by comparing results of TPS calculations and dosimetric measurements for $6 \mathrm{MV}$ beam energy. The TiLOOP mesh used in breast surgery did not affect dose calculations for RT. When the target to be irradiated is close to the mesh, no significant dose difference was found with or without mesh. In addition, there were no metallic artifacts on CT image. Therefore, the quality of the CT image was not affected by the TiLOOP Bra mesh, and it is not necessary to correct the artifact and change the HU value.

Peer-review: Externally peer-reviewed.

Conflict of Interest: None declared.

Ethics Committee Approval: This study was conducted in accordance with local ethical rules.

Financial Support: None declared.

Authorship contributions: Concept - S.Ç.D.; Design S.Ç.D.; Supervision - S.Ç.D.; Materials - S.Ç.D., A.T.; Data collection \&/or processing - S.Ç.D., A.T.; Analysis and/or interpretation - S.Ç.D., A.T.; Literature search - S.Ç.D., S.T., P.E.; Writing - S.Ç.D., S.T., P.E.; Critical review - S.Ç.D., S.T., P.E.

\section{References}

1. Dieterich M, Paepke S, Zwiefel K, Dieterich H, Blohmer J, Faridi A, et al. Implant-based breast recon- struction using a titanium-coated polypropylene mesh (TiLOOP Bra): a multicenter study of 231 cases. Plast Reconstr Surg 2013;132(1):8-19.

2. AAPM. Tissue inhomogeneity corrections for megavoltage photon beams, Report No. AAPM-85. AAPM Task Group No.65 of the Radiation Therapy Committee. Madison, WI: Medical Physics Publishing; 2004.

3. Catlı S, Tanır G. Experimental and Monte Carlo evaluation of Eclipse treatment planning system for effects on dose distribution of the hip prostheses. Med Dosim 2013; 38(3):332-6.

4. Çatli S. High-density dental implants and radiotherapy planning: Evaluation of effects on dose distribution using pencil beam convolution algorithm and Monte Carlo method. Journal of Applied and Clinical Medical Physics 2015;16(5):46-52.

5. Lin SY, Chu TC, Lin JP, Liu MT. The effect of a metal hip prosthesis on the radiation dose in therapeutic photon beam irradiations. Appl Radiat Isot 2002;57(1):17-23.

6. Biggs PJ, Russell MD. Effect of femoral head prosthesis on megavoltage beam radiotherapy. Int J Radiat Oncol Biol Phys 1988;14(3):581-6.

7. Carolan M, Dao P, Fox C, Metcalfe P. Effect of hip prostheses on radiotherapy dose. Australas Radiol 2000;44(3):290-5.

8. Mesbahi A, Nejad FS. Dose attenuation effect of hip prostheses in a 9-MV photon beam: commercial treatment planning system versus Monte Carlo calculations. Radiat Med 2007; 25(10):529-35.

9. Shimozato T, Yasui K, Kawanami R, Habara K, Aoyama Y, Tabushi K, et al. Dose distribution near thin titanium plate for skull fixation irradiated by a $4-\mathrm{MV}$ photon beam. J Med Phys 2010;35(2):81-7.

10. Camacho C, Pujades MC, Perez-Calatayud J, Lliso F, Carmona V, Tormo A, et al. Impact of the Tiloop Bra mesh in CT images and dose delivery in breast radiotherapy. J Appl Clin Med Phys 2012;13(2):3667.

11. Cho S, Goh Y, Kim C, Kim H, Jeong JH, Lim YK, et al. Dosimetric Impact of Ti Mesh on Proton Beam Therapy. Progress in Medical Physics 2017; 28(4):144-8.

12. Patone H, Barker J, Roberge D. Effects of neurosurgical titanium mesh on radiation dose Med Dosim 2006; 31(4): 298-301.

13. Rakowski JT, Chin K, Mittal S. Effects of titanium mesh implant on dosimetry during gamma knife radiosurgery J Appl Clin Med Phys 2012;13(5):54-61.

14. ICRU Report 24. Determination of Absorbed Dose in Patient Irradiated by Beams of X or Gamma Rays in Radiotherapy. ICRU;1976.

15. Das IJ, Kahn FM. Backscatter dose perturbation at high atomic number interfaces in megavoltage photon beams. Med Phys 1989;16(3):367-75. 